\title{
Prevalence and ultrasound patterns of testicular adrenal rest tumors in adults with congenital adrenal hyperplasia
}

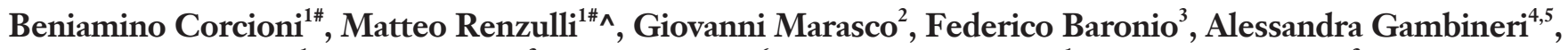 \\ Domenico Ricciardi $^{1}$, Rita Ortolano ${ }^{3}$, Davide Farina ${ }^{6}$, Caterina Gaudiano ${ }^{1}$, Alessandra Cassio ${ }^{3}$, \\ Uberto Pagotto $^{4,5}$, Rita Golfieri ${ }^{1}$
}

${ }^{1}$ Department of Radiology, IRCCS Azienda Ospedaliero-Universitaria di Bologna, Bologna, Italy; ${ }^{2}$ Department of Medical and Surgical Sciences, S. Orsola Hospital, University of Bologna, Bologna, Italy; ${ }^{3}$ Pediatric Unit, Department of Medical and Surgical Sciences, S. Orsola Hospital, University of Bologna, Bologna, Italy; ${ }^{4}$ Division of Endocrinology and Diabetes Prevention and Care, IRCCS Azienda Ospedaliero-Universitaria di Bologna, Bologna, Italy; ${ }^{5}$ Department of Medical and Surgical Sciences (DIMEC), Alma Mater Studiorum University of Bologna, Bologna, Italy; ${ }^{6}$ Department of Medical and Surgical Specialties, Radiological Sciences, and Public Health, University of Brescia, Brescia, Italy

Contributions: (I) Conception and design: M Renzulli, B Corcioni, G Marasco, R Golfieri; (II) Administrative support: B Corcioni, M Renzulli, C Gaudiano, R Golfieri; (III) Provision of study materials or patients: F Baronio, B Corcioni, R Ortolano, A Cassio, A Gambineri, U Pagotto, M Renzulli, R Golfieri; (IV) Collection and assembly of data: D Ricciardi, M Renzulli, B Corcioni, G Marasco, D Farina, F Baronio, R Ortolano, A Gambineri, A Cassio, U Pagotto; (V) Data analysis and interpretation: M Renzulli, B Corcioni, G Marasco, D Farina; (VI) Manuscript writing: All authors; (VII) Final approval of manuscript: All authors.

"These authors contributed equally to this work.

Correspondence to: Matteo Renzulli, MD. Department of Radiology, IRCCS Azienda Ospedaliero-Universitaria di Bologna, Bologna, Italy. Email: matteo.renzulli@aosp.bo.it.

Background: Testicular adrenal rest tumors (TARTs) are benign neoplasms affecting patients with congenital adrenal hyperplasia (CAH). The prevalence of TART in adult patients with CAH is not well known. Ultrasonography (US) is the main tool for diagnosing TART and the role of contrast-enhanced US (CEUS) is never investigated. The aim of this study was to evaluate the TART prevalence in adults with $\mathrm{CAH}$, by stratifying patients according to disease phenotype and assessing the diagnostic performance of US, color Doppler (CD) US and CEUS.

Methods: Male patients $>16$ years old with certain diagnosis of CAH who underwent US for TARTs, between December 2015 and September 2019 were prospectively enrolled. The control group included patients without CAH affected by testicular lesions at US other than TARTs.

Results: TARTs were identified in 16 of 52 patients (31\%), of whom 15 (93.8\%) displayed the salt-wasting (SW) form $(\mathrm{P}<0.001)$. The prevalence of TARTs in patients with the $\mathrm{SW}$ form was $54 \%$. One patient with the non-classic (NC) form (6\%) showed TART (likely the first documented case). The mean age of patients upon detection of TARTs was significantly younger compared with the control group $(\mathrm{P}<0.001)$; moreover, TARTs were bilateral in $15 / 16$ patients $(93.8 \% ; \mathrm{P}<0.001)$ and the largest lesion was more frequent in the medium third of testis in the TART group (87.5\%), statistically different from the control group ( $\mathrm{P}=0.013)$.

Conclusions: TARTs almost exclusively affected patients with the SW form of CAH. Age at diagnosis and bilateralism are useful factors for achieving a correct diagnosis of TARTs in CAH adult patients.

Keywords: Testicular adrenal rest tumors (TARTs); congenital adrenal hyperplasia (CAH); ultrasonography (US)

Submitted Jun 15, 2020. Accepted for publication Oct 16, 2020.

doi: $10.21037 /$ tau-20-998

View this article at: http://dx.doi.org/10.21037/tau-20-998

\footnotetext{
^ ORCID: 0000-0002-1311-5670.
} 


\section{Introduction}

Adrenal rest tumors represent a rare group of benign neoplasms that originate from cells with a biological profile similar to adrenal cells but are located in extra-adrenal sites (1-6). Among these tumors, testicular adrenal rest tumors (TARTs) are the most studied neoplasm due to their higher frequency.

TARTs were described for the first time by Wilkins in 1940 (7). Regardless of the different etiological theories (8-10), adrenocorticotropic hormone (ACTH) has been recognized as the most important factor promoting the development of TARTs $(1,11,12)$. Several diseases are characterized by an increase of ACTH; however, TARTs are discovered to exist almost exclusively in patients affected by congenital adrenal hyperplasia $(\mathrm{CAH})$, excluding only rare cases (13-15).

$\mathrm{CAH}$ is a rare autosomal recessive disease that is generally (over $95 \%$ of all cases) caused by mutations in CYP21A2, the gene encoding the adrenal enzyme 21-hydroxylase (21OHD). CAH due to $21 \mathrm{OHD}$ deficiency is a continuum of disease severity that depends on the residual enzyme activity. The residual enzyme activity, which mainly depends on the genotype alterations (16), produces two different phenotypical categories defined as classic forms: (I) the salt-wasting (SW) form, which is the most severe type and is characterized by cortisol and aldosterone deficiencies due to absent residual enzyme activity; and (II) the simple virilizing (SV) form, in which the residual enzyme activity (1-2\%) generally produces a sufficient aldosterone concentration but low cortisol and strongly elevated androgen concentrations. The incidence of classic CAH is estimated at 1:10,000 to $1: 20,000$ births worldwide $(15,17)$. The non-classic (NC) form of CAH is characterized by a residual enzyme activity of $30-50 \%$, only slightly elevated androgens, and generally sufficient cortisol and aldosterone concentrations. Therefore, it is considered the mildest form of CAH. The NC form is much more common, with an estimated prevalence of $1 / 1,000$ births worldwide (18). There are other subtypes of CAH in which TARTs have been proven to occur, but these forms are extremely rare (19).

Different studies have demonstrated the possibility of adjusting hormonal replacement therapy for containing or even inducing the regression of TARTs in the early stages (20-23). Therefore, the correct diagnosis of TARTs is essential in the early stages of the disease. Furthermore, a correct diagnosis is also important for preventing misdiagnosis and avoiding unnecessary testicular surgery (17,24-27).

Physical examination may detect a TART as a palpable mass when the lesion has a diameter of at least $2 \mathrm{~cm}$, with a detection rate between $5 \%$ and $35 \%(28,29)$. Ultrasonography (US) demonstrated a detection rate of 89-94\%, even for smaller lesions (30-32).

TARTs have a prevalence of $37 \%$ in patients with CAH. Unfortunately, currently, there are no robust data concerning the prevalence of TARTs in adult patients with $\mathrm{CAH}$ due to the lack of dedicated studies with large populations. A recent review reported the TART prevalence in adults affected by $\mathrm{CAH}$ to be $46 \%$ compared with $25 \%$ in studies including only children (1). However, this result is affected by several limitations: (I) not every enrolled study stratified the patients according to $\mathrm{CAH}$ phenotype; (II) the studies analyzed in the review were not focused exclusively on adults; and (III) the NC subtype form of CAH was often not included.

The aim of this study was to assess the prevalence of TARTs in adults with CAH who are already under hormone therapy by stratifying the population according to the three different CAH phenotypes (SW, SV, and NC forms). The secondary aim was to assess the diagnostic performance of US, color Doppler (CD) US, and contrast-enhanced US (CEUS) in the evaluation of testicular lesions.

We present the following article in accordance with the STROBE reporting checklist (available at http://dx.doi. org/10.21037/tau-20-998).

\section{Methods}

The local institutional review board (No. RIF.CE: 4882) approved this prospective study, and written informed consent was obtained from all patients. This study was conducted according to the Declaration of Helsinki (as revised in 2013) for clinical studies.

Male patients with a certain diagnosis of CAH who underwent US for TARTs at our institution between December 2015 and September 2019 were eligible for this prospective study.

In all patients, the diagnosis of $\mathrm{CAH}$ was made on symptoms and laboratory tests and was confirmed by mutation analysis (33).

The inclusion criteria of this prospective study were as follows: (I) patients $>16$ years old; and (II) patients under treatment with hormonal replacement therapy based on glucocorticoids. Serum levels of ACTH were measured in all patients. ACTH was used as the hallmark in the evaluation 
of hormonal control, and the adequate control of $\mathrm{CAH}$ was defined as ACTH serum levels less than $50 \mathrm{pg} / \mathrm{mL}$.

\section{Image technique and analysis}

All the enrolled patients were evaluated with the same US machine: Canon-Toshiba Aplio 500 ${ }^{\mathrm{TM}}$ (Ōtawara, Kanto, Japan).

Both testicles were evaluated, and the following features were recorded on a dedicated database:

(I) Testicle echogenicity, recorded as homogeneous or heterogeneous;

(II) The entire didymus volume, calculated by applying the formula for an ellipsoid (length $\times$ width $\times$ thickness $\times 0.52$ );

(III) Additional didymal and scrotal features such as varicocele or hydrocele;

(IV) Presence or absence and number of lesions. In cases with multiple lesions, each was evaluated as a single lesion;

(V) Lesion's location, recorded as in the upper, medium, or inferior third of the didymus;

(VI) Testicular lesion size measured as the maximum diameter;

(VII) Characteristics of testicular lesions: echogenicity, margins, vascularization on CD-US and CEUS.

The echogenicity of the lesion was determined as hypo-, iso-, or hyper-echoic or heterogeneous.

The margins of the lesions were recorded as oval or irregular.

In the presence of testicular lesions, CD-US and CEUS were performed.

Two different patterns were recognized on the CD-US: (I) high, uniform, and diffuse signals or peripheral rim of increased signals; and (II) no difference in signals with the contiguous normal testicular parenchyma. The same probe used for CD-US was used for CEUS in a low mechanical index (range, 0.04-0.1) after the injection of $4.8 \mathrm{~mL}$ of contrast agent (SonoVue ${ }^{\circledast}$, Bracco, Milano, Italy), followed by $10 \mathrm{~mL}$ of $0.9 \%$ saline. Contrast enhancement was evaluated for at least 2 minutes after the SonoVue injection, and different phases of contrast kinetics were stored as video clips. Low mechanical index mode and the gray scale imaging picture were simultaneously recorded on a split screen. The CEUS results were interpreted by comparing the brightness of the lesion to the surrounding testicular tissue after the injection of the contrast agent, with three different patterns identified: (I) hyperenhancement, (II) isoenhancement, and (III) hypoenhancement.

The study design included the use of a control group of patients without $\mathrm{CAH}$ who were affected by testicular lesions other than TARTs. This control group was retrospectively selected among patients with testicular lesions of similar dimensions who underwent US examination at our institution. The same demographic information, clinical characteristics, and US features of the control group were recorded as those for the CAH patient population.

\section{Statistical analysis}

Data were reported as counts and percentages for categorical variables and the median and inter-quartile range for continuous variables. Categorical variables were compared by the Chi-square or Fisher's exact tests, as appropriate. Continuous variables were compared with the Kruskal-Wallis test. Comparisons for demographics and general clinical characteristics were performed between groups of patients with CAH with and without TARTs and between patients with TARTs compared with consecutive patients affected by testicular lesions other than TARTs.

Univariate and multivariate logistic regressions were performed to assess the demographic, pathological, and testicular ultrasound characteristics associated with TART diagnosis. The results were reported as odds ratios (ORs) with $95 \%$ confidence intervals (95\% CIs). An OR with the entire $95 \%$ CI less than 1 indicated that the covariate reduced the probability of a TART diagnosis. Conversely, when the OR and entire $95 \% \mathrm{CI}$ were greater than 1 , the covariate increased the abovementioned risk. An OR with a $95 \% \mathrm{CI}$ equal to 1 indicated that the covariate did not significantly influence this probability. The results obtained for the clinical endpoints from the multivariate analysis, in the presence of two or more covariates influencing the risk, were translated in graphic form through the use of special nomograms for logistic regression. The probability values are two-sided; a probability value of less than 0.05 was considered to indicate statistical significance. All computations were performed using SPSS 13 (SPSS Inc., Chicago, IL, USA).

\section{Results}

Fifty-two patients with CAH satisfied the inclusion criteria and were enrolled in this study. The median [range] age of this patient population was 22.5 [16-48] years. 
Table 1 Demographics and clinical characteristics of patients enrolled in the study

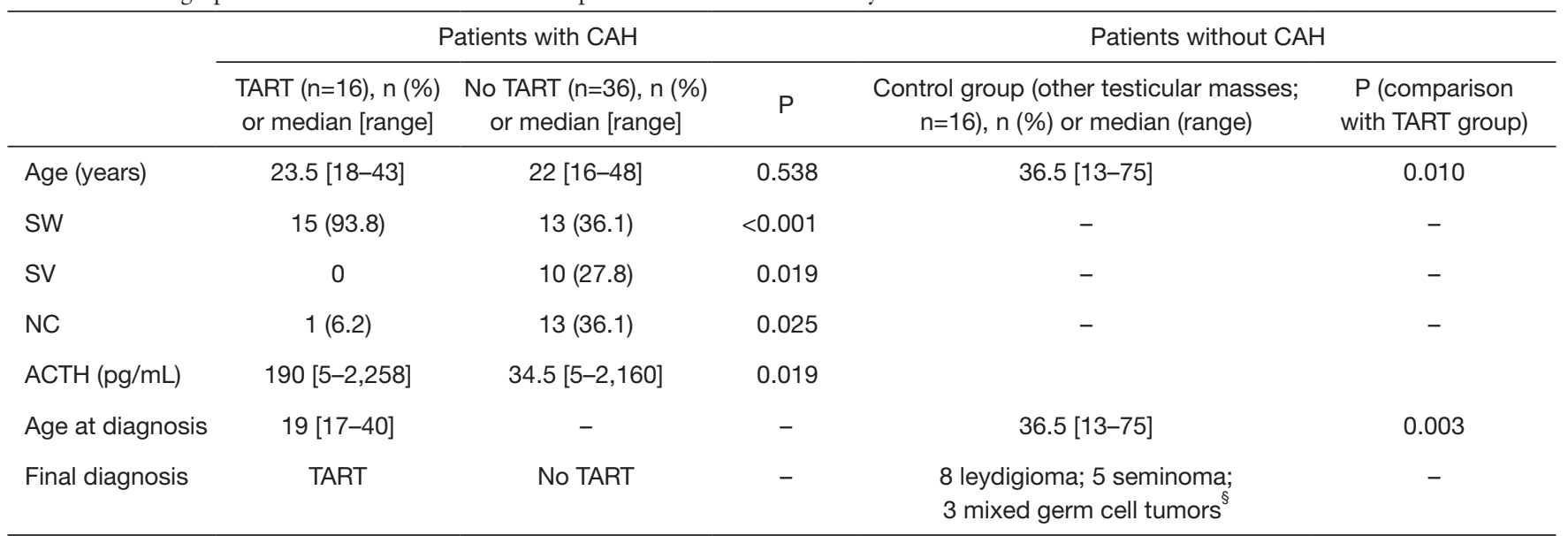

§, Mixed germ cell tumors: seminomatous and non-seminomatous germ-cell tumors. CAH, congenital adrenal hyperplasia; TART, testicular adrenal rest tumor; SW, salt-wasting; SV, simple virilizing; NC, non-classic; ACTH, adreno-cortico-tropic hormone.

The control group consisted of 16 patients, including $8(50 \%)$ affected by leydigioma, 5 (31\%) affected by seminoma, and $3(19 \%)$ affected by mixed germ-cell tumors (seminomatous and non-seminomatous germ-cell tumors). The median [range] age of the control group was 36.5 [13-75] years, which was statistically older than that of the $\mathrm{CAH}$ patient population $(\mathrm{P}<0.001)$. The demographic and clinical characteristics of the enrolled patients are detailed in Table 1.

Twenty-eight patients with CAH (54\%) had the classic SW form of CAH, 10 patients (19\%) had the SV form, and 14 patients $(27 \%)$ had the NC form.

Focal lesions compatible with TARTs were identified in 16 of 52 patients (31\%), of whom 15 (93.8\%) patients had the SW form $(\mathrm{P}<0.001)$. The prevalence of TARTs was $54 \%$ in patients with the SW form. Only 1 patient with the $\mathrm{NC}$ form $(6 \%)$ demonstrated TARTs. No TARTs were detected in patients with the SV form.

The median [range] age at the detection of TARTs was 19 [17-40] years, which was statistically younger than that of the control group $\{36.5$ [13-75] years; $\mathrm{P}=0.003\}$.

In the CAH patient population, the ACTH values were significantly higher (median $190 \mathrm{pg} / \mathrm{mL}$ ) in the TART group than in the non-TART group (median $34.5 \mathrm{pg} / \mathrm{mL}$; $\mathrm{P}=0.019)$.

The ultrasound features of patients with TARTs and of the control group with other testicular masses are detailed in Table 2.

There were no statistical differences between the two groups in terms of testicular appearance, volume, and extradidymal findings (Figure 1).

Overall, there were 51 lesions compatible with TARTs in the CAH population: 8 (50\%) patients had two TARTs and the remaining $50 \%$ had three or more lesions. No patients had only one nodule. There was a total of 17 lesions in the control group, of which only one patient presented with two lesions. This latter figure was significantly different compared with the TART group $(\mathrm{P}<0.001)$.

In the TART group, the lesions were bilateral in $15 / 16$ patients (93.8\%), differently from the control group $(\mathrm{P}<0.001)$. There were no differences in the incidence of lesions in the left didymus between the two groups. Conversely, the incidence of lesions in the right testis was significantly higher in the TART population than in the control group $(\mathrm{P}=0.002)$.

The largest lesion was significantly more frequently located in the medium third of the didymus in the TART group (87.5\%) than in the control group ( $\mathrm{P}=0.013)$.

In both groups, the most common US appearance of lesions was hypoechogenicity, which did not differ significantly between groups. No differences were found between the two populations in terms of lesion dimension and morphology or in the appearance on CD-US or CEUS.

The results of multivariate analyses (Table 3) for number of right testicular lesions and age at diagnosis are graphically reported in nomogram (Figure 2).

\section{Discussion}

The vast majority of TARTs are discovered in patients 
Table 2 Ultrasound features of patients with TARTs compared to other testicular masses

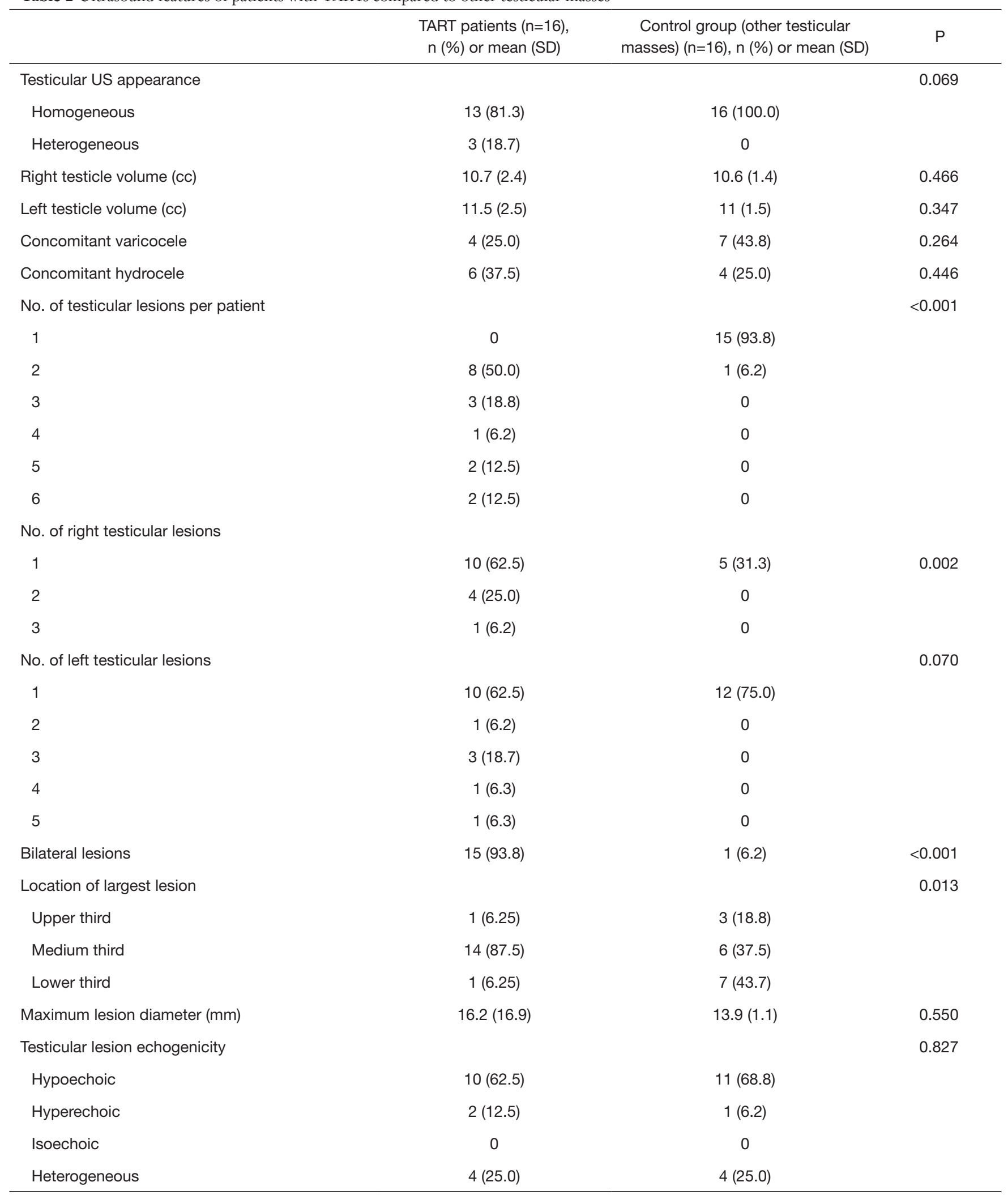

Table 2 (continued) 
Table 2 (continued)

\begin{tabular}{lcc}
\hline & $\begin{array}{c}\text { TART patients }(\mathrm{n}=16), \\
\mathrm{n}(\%) \text { or mean }(\mathrm{SD})\end{array}$ & $\begin{array}{c}\text { Control group (other testicular } \\
\text { masses) }(\mathrm{n}=16), \mathrm{n}(\%) \text { or mean (SD) }\end{array}$ \\
\hline Testicular lesion morphology & $8(50.0)$ & $9(56.3)$ \\
Oval & $8(50.0)$ & $7(43.7)$ \\
Irregular & $12(75.0)$ & $11(68.8)$ \\
Intra-lesional signal on CD & & $13(81.2)$ \\
CEUS appearance & $13(81.2)$ & $3(18.8)$ \\
Hyperenhancement & $3(18.8)$ & - \\
Isoenhancement & - & 0.694 \\
Hypoenhancement & & 1 \\
\hline
\end{tabular}

TART, testicular adrenal rest tumor; SD, standard deviation; US, ultrasound; CD, color Doppler; CEUS, contrast-enhanced US.
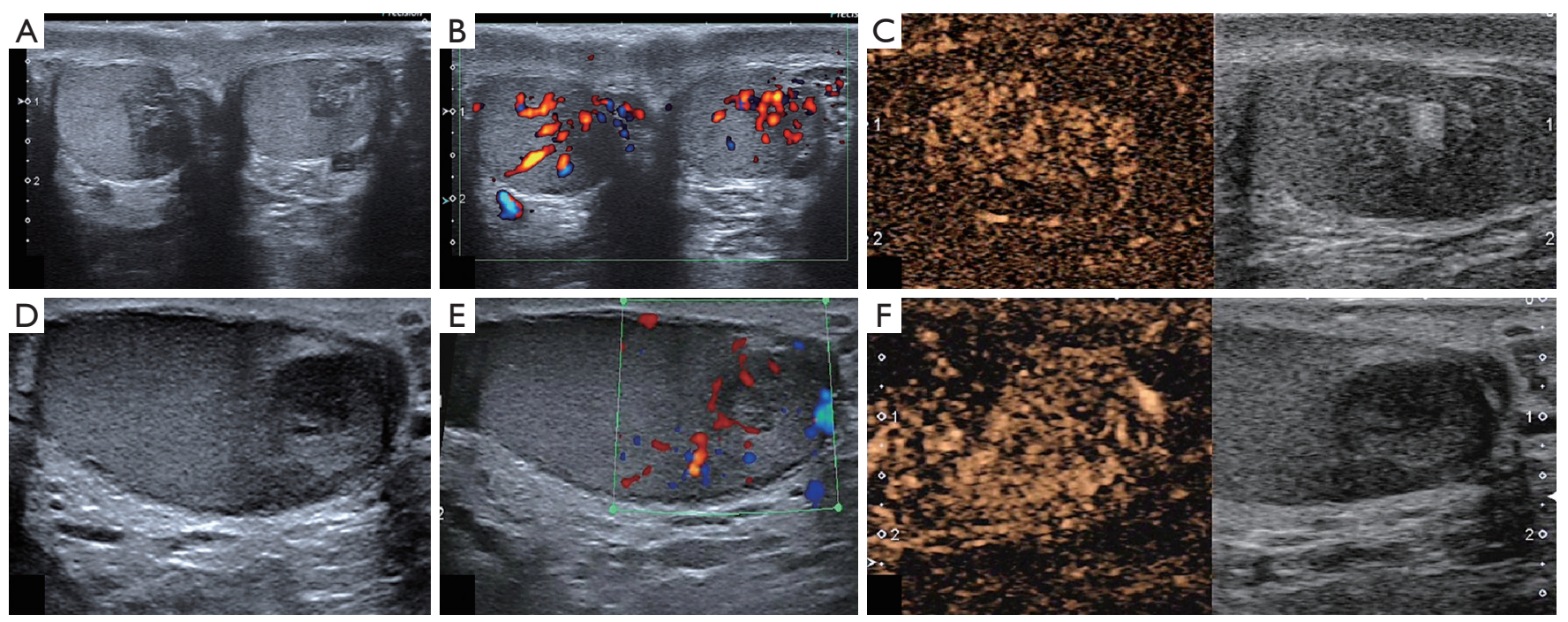

Figure 1 This figure demonstrates two different patients, one affected by CAH with TARTs (A,B,C) and one with a testicular nonseminomatous germ-cell tumor (D,E,F). The lesions showed the same imaging features, such as a hypoechoic appearance on B-mode (A,D), intralesional signal on CD (B,E), and hypervascularity on CEUS (C,F). The only differences between the two cases were the bilateralism of lesions in the patient with TARTs (A) and the different location: in the middle third for TARTs (A) and in the inferior third in the nonseminomatous germ-cell tumor (D). CAH, congenital adrenal hyperplasia; TART, testicular adrenal rest tumor; CD, color Doppler; CEUS, contrast-enhanced ultrasonography.

affected by CAH (13-15). Unfortunately, currently, there are no robust data concerning the prevalence of TARTs in adult patients with CAH due to the lack of dedicated studies with significant series populations.

The present study population was comprised of 52 adult patients with CAH. The largest series in the literature were those of Engels et al. (34) who enrolled 141 adult men with CAH, and of Finkielstain et al. (12) who enrolled 244 patients. However, the study of Engels et al. (34) involved 14 centers in six different European countries whereas that of Finkielstain et al. (12) included both children [170] and adults [74] from 36 states in the United States of America and from Puerto Rico, Canada, Ecuador, Turkey, and the Dominican Republic. Therefore, our study 
Table 3 Uni- and multi-variate analyses for evaluating factors associated with TART diagnosis during testicular lesion evaluation

\begin{tabular}{|c|c|c|c|c|}
\hline & \multicolumn{2}{|c|}{ Univariate analysis } & \multicolumn{2}{|c|}{ Multivariate analysis } \\
\hline Age at diagnosis (years) & $0.872(0.793-0.959)$ & 0.005 & $0.850(0.731-0.989)$ & 0.035 \\
\hline Right testicular volume (cc) & $1.017(0.708-1.461)$ & 0.926 & & \\
\hline Left testicular volume (cc) & $1.113(0.786-1.575)$ & 0.564 & & \\
\hline Concomitant hydrocele & $1.8(0.394-8.215)$ & 0.448 & & \\
\hline Number of right testicular lesions & $25.104(2.749-229.271)$ & 0.004 & $82.608(2.690-2537.06)$ & 0.012 \\
\hline Number of left testicular lesions & $1.017(0.957-1.081)$ & 0.589 & & \\
\hline \multicolumn{5}{|l|}{ Testicular lesion ultrasound echogenicity } \\
\hline Isoechoic & - & - & & \\
\hline Heterogeneous & $1.1(0.215-5.609)$ & 0.909 & & \\
\hline Testicular lesion irregular morphology & $1.288(0.320-5.169)$ & 0.723 & & \\
\hline Presence of intra-lesion CD signal & $1.363(0.289-6.415)$ & 0.695 & & \\
\hline Hyperenhancement on CEUS & $1(0.169-5.903)$ & 1 & & \\
\hline Location of maximum diameter testicular lesions & $0.485(0.142-1.657)$ & 0.248 & & \\
\hline
\end{tabular}

TART, testicular adrenal rest tumor; OR, odds ratio; CI, confidence interval; CD, color Doppler; CEUS, contrast-enhanced ultrasonography.

population was representative because it was prospectively recruited from a single tertiary center and included only adult patients.

Our study population had a median age of 22.5 years, in line with those reported by Engels et al. (34) and Finkielstain et al. (12). In fact, the mean ages reported by those authors were 28 and 31 years, respectively, in adults. Moreover, in the present study, the median age at the diagnosis of TARTs was 19 years, which was significantly lower than that of the control group of patients without CAH who were affected by testicular masses other than TARTs (36.5 years; $\mathrm{P}=0.003$ ). This result, which was difficult to determine in other studies $(12,34)$, appears important for identifying at what age a patient with $\mathrm{CAH}$ should undergo US surveillance for TARTs.

In the present study, the TART prevalence was $31 \%$, and the lesions were discovered in patients with the $S W$ form of $\mathrm{CAH}$ in the vast majority of cases $(94 \% ; \mathrm{P}<0.001)$. The prevalence of TARTs was $54 \%$ in patients with the SW form, which represented the most common form of CAH in our sample (54\%). No TARTs were detected in patients with the SV form. Only 1 patient with the NC form (6\%) displayed TARTs. The overall prevalence of TARTs in our series $(31 \%)$ was lower than that of other studies on adult populations, such as the series of Finkielstain et al. (12) who reported a prevalence of $44 \%$. This difference was only apparent because Finkielstain et al. (12) calculated this percentage as the number of TARTs only in "Classic men" $(\mathrm{SW}+\mathrm{SV})$. Therefore, if we calculate the percentage in the same manner, our prevalence was $39.5 \%$ in patients with only classic forms of $\mathrm{CAH}$, similar to the abovementioned series. In another study by Dumic et al. (35), the overall TART prevalence was $29 \%$, similar to our series. However, the study population by Dumic et al. (35), was composed of both children and adult patients. In fact, the prevalence of TART exclusively in adults was $44 \%$, but the percentage of SW forms was not reported in adults.

The absence of TARTs in the SV form of CAH in our series is in line with the vast majority of published series (34,36-38). Furthermore, in our series, only one patient with the NC form presented with TARTs. Although the $\mathrm{NC}$ form of $\mathrm{CAH}$ has a high prevalence in the general 
Probability of TART diagnosis

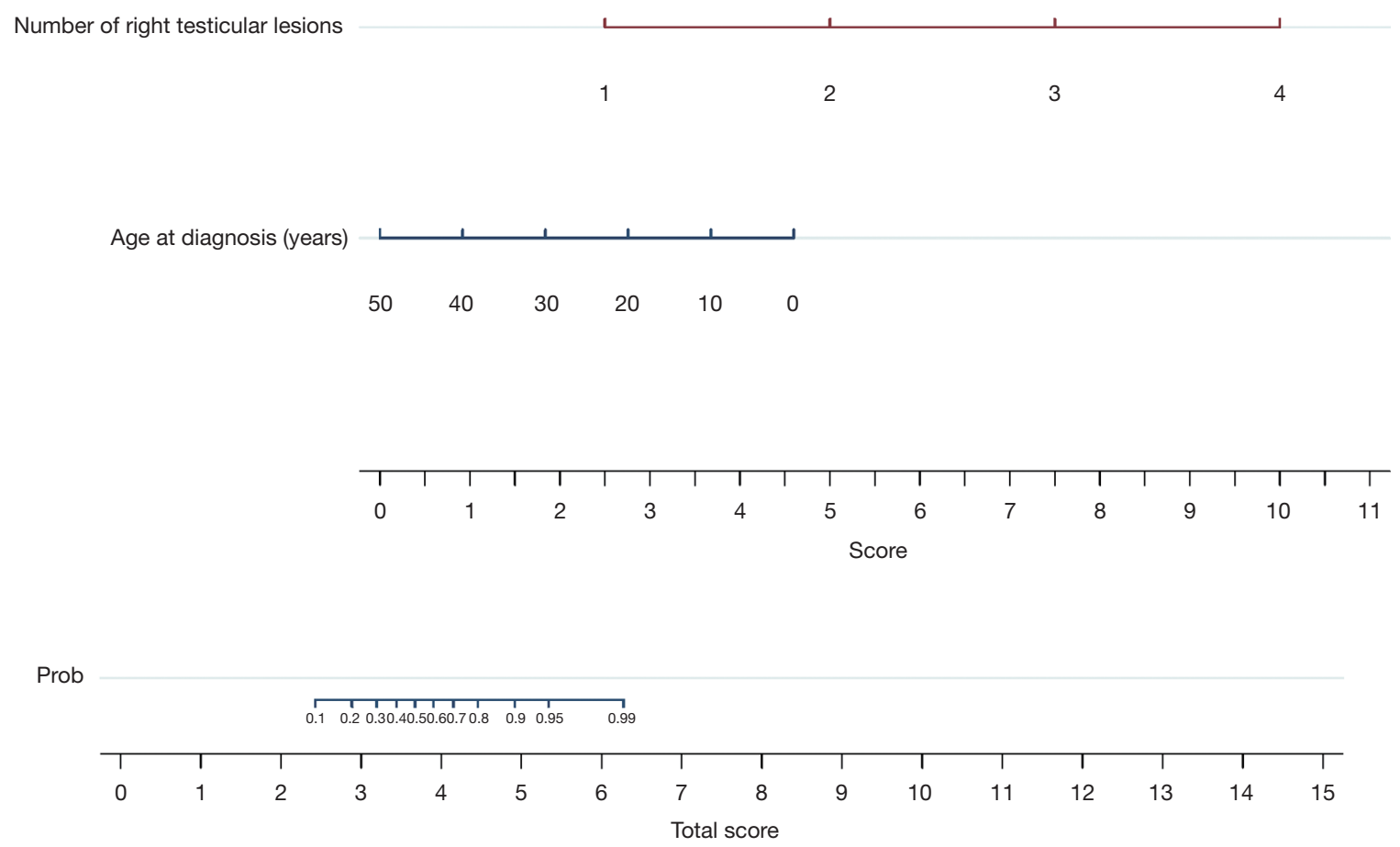

Figure 2 Nomogram. Nomogram reporting a probability score for TART diagnosis expressed by the addition of single score for the number of right testicular lesions and the age at diagnosis. The number of right testicular lesions was associated with a score of 1-4, while the age at diagnosis varied from $0-4.5$. TART, testicular adrenal rest tumor.

population, with a rate of 1 per 1,000 births, TARTs are reported to be exceptionally rare in these individuals. In fact, to the best of our knowledge, our case represents the sixth case of TARTs associated with NC in published series (1). In particular, Falhammar et al. (18), Engels et al. (34), and Kocova et al. (39) described the other five incidences of TARTs in patients with the NC form of CAH. However, the phenotype of these patients was not detailed, and based on mutation status, these patients might also belong to the more affected classic form of CAH. Conversely, our case was proven to be an $\mathrm{NC}$ form of $\mathrm{CAH}$ (this may be the first case of a patient proven to have the NC form of CAH affected by TARTs). It is likely that data concerning the prevalence of TARTs in the NC form of CAH could be underestimated due to the lack of specific studies on large populations. Moreover, the NC form of CAH often remains unknown because it is characterized by only a few symptoms or is completely asymptomatic, and there is no actual screening test for this form. Conversely, the high frequency of TARTs in the SW form compared to the other forms of CAH may suggest the prominent role of the disease severity. In fact, compared to the other parameters such as age at detection, modality of diagnosis, and hormonal control, several studies have indicated that the SW phenotype is the main risk factor for developing TARTs $(1,12,17,27,28)$.

In our $\mathrm{CAH}$ patient population, ACTH values were significantly higher in the TART group than in the nonTART group $(\mathrm{P}=0.019)$. This result supports the current perception that TARTs occur more frequently in patients affected by more severe forms of $\mathrm{CAH}$ because these patients are exposed to higher levels of ACTH, which is a possible causative factor for TART development $(29,38,40,41)$. The first etiological theory of TARTs assumes that these lesions derived from ectopic adrenal cells and migrated to the gonads in fetal life, and their growth is under control of ACTH and angiotensin II $(42,43)$. The second etiological theory sustains that TARTs may originate from pluripotent fetal Leydig cells that have ACTH and 
luteinizing hormone receptors (10). In both etiological theories of TART, ACTH plays an important rule, justifying our results.

There were 51 overall lesions compatible with TARTs in the CAH population in our series: $8(50 \%)$ patients had two TARTs and the remaining $50 \%$ had three or more lesions. No patients had only one nodule. There were 17 testicular lesions in the control group of no-CAH patients with testicular lesions other than TART, and only one patient presented two lesions, differently from the TART group $(\mathrm{P}<0.001)$. The multiplicity of lesions per testis (more than one for didymus) has been described in the original series by Stikkelbroeck et al. (31), Wang et al. (44), and Chihaoui et al. (45), but with frequencies of $19 \%, 16 \%$, and $25 \%$, respectively, which were all lower than ours. According to the actual state of knowledge about TART histopathology, TARTs are thought to arise as small multiple nodules that tend progressively to coalesce into a single nodule. Thus, different numbers of nodules per testis are considered a reflection of a different pathological stage (31). The high frequency of the multiplicity of nodules per testis likely indicates that our cohort of patients was composed mainly of subjects with an early-intermediate stage of CAH disease.

In the present series, in the TART group, the lesions were bilateral in the vast majority of patients (93.8\%), unlike that observed in patients without $\mathrm{CAH}$ who were affected by testicular tumors other than TART $(\mathrm{P}<0.001)$. There were no differences in the incidence of lesions in the left didymus between the two groups. Conversely, the incidence of TARTs in the right testis was significantly higher in the TART population than in the control group $(\mathrm{P}=0.002)$. Bilateralism is traditionally considered one of the best clues in the diagnosis of TARTs. In fact, different published series reported bilateral nodules compatible with TART in all of their cases $(10,30,46)$. Nevertheless, different series have reported that bilateralism was not present in all cases $(17,20,28,34)$. In particular, the lowest percentage of bilateralism (31\%) was detected by Claahsenvan der Grinten et al. (20). It is interesting to note that all the nodules detected by Claahsen-van der Grinten et al. (20) were less than $10 \mathrm{~mm}$ in size. In our case series, the mean maximum diameter was greater than $10 \mathrm{~mm}$. Our results sustain the hypothesis that TARTs may always have a bilateral process, but in patients with early stages of TARTs, indicated by the small dimension of the lesions, synchronous contralateral lesions may be too small to be detected.

The largest lesions were significantly more frequently located in the medium third of the didymus, near the rete testes, in the TART group (87.5\%) compared with the control group $(\mathrm{P}=0.013)$. This is a hallmark of TARTs that is reported constantly in the literature $(1,29,30)$. A single case of our series displayed involvement of the epididymis by a nodule compatible with TARTs. Although no pathological proof of its nature was achieved, the epididymis has been described as an unusual location for TARTs (2).

In the vast majority of our cases, the TARTs appeared hypoechoic on US, with intra-lesional signals on the CDUS and hypervascularization on CEUS. However, these features were not specific for TARTs compared with the control group. Furthermore, no statistical differences were found in terms of didymal and extra-didymal features in patients with CAH and TARTs in respect to the subjects without CAH who were affected by testicular lesions other than TARTs. These data do not differ significantly from other original studies $(28,31,44)$.

The role of CD-US in TART characterization is controversial due to a great discordance present in the literature. Several authors $(28,31,34)$ failed to identify an increased vascular signal on CD-US in their series, unlike other experiences $(30,44,45)$.

Differently from CD-US, there are no published series exploring the role of CEUS in TARTs. Therefore, our results are the first that reported imaging findings of CEUS in patients affected by TARTs.

Parenchymal echogenicity of testes has been reported in a few studies, such as those by Bouvattier et al. (17) and Cabrera et al. (47), with results very similar to ours.

One unicity of the present study is the comparison of $\mathrm{CAH}$ patients with TARTs with non-CAH subjects with testicular lesions other than TARTs. Finally, none of the US, CD-US, and CEUS features of the lesions and of the didymus or extradidymus showed statistically significant differences between TART and non-TART testicular lesions. Therefore, in our opinion, the imaging figures are helpful for detecting TARTs in high-risk patients such as adults affected by CAH, rather than performing a differential diagnosis.

Another novelty of this study is the introduction of the nomogram for the risk estimation of TARTs by the radiologist based on the number of right testicular lesions and age at diagnosis. The nomogram is a two-dimensional diagram that allows the approximate graphical calculation of a function, such as a multivariate analysis. These graphical instruments are easy to use and enable rapid calculation of the pathological risks for a single patient in the era of tailored medicine. 
This study is affected by several limitations. First, a histological diagnosis of suspected TART lesions in $\mathrm{CAH}$ patients was not achieved. However, the biopsy is not indicated in the clinical suspect of TARTs. The main limitation is the difficulty in recruiting a large study population, due the rarity of the disease. However, this work could represent a good model for a single tertiary center study of the prevalence of TARTs in CAH. Furthermore, due to the limited sample size of our study, the multivariate analysis is affected by an overfitting variables bias. However, the results of the present multivariate analysis should be interpreted with caution but are still valuable for addressing further larger studies in order to validate our results.

In conclusion, TARTs affected approximately onethird of the adult patients with $\mathrm{CAH}$, principally those affected by the SW form. The age at diagnosis and the right testicular lesions appear very useful figures to achieve a correct diagnosis of TARTs in CAH patients. No specific US figures were demonstrated to be helpful in differential diagnosis with other testicular lesions; therefore, US is mandatory only for lesion detection. If our results are confirmed by other studies, it will be possible to plan US surveillance programs in patients with the $S W$ form of $\mathrm{CAH}$ starting from adulthood.

\section{Acknowledgments}

Funding: None.

\section{Footnote}

Reporting Checklist: The authors have completed the STROBE reporting checklist. Available at http://dx.doi. org/10.21037/tau-20-998

Data Sharing Statement: Available at http://dx.doi. org/10.21037/tau-20-998

Peer Review File: Available at http://dx.doi.org/10.21037/ tau-20-998

Conflicts of Interest: All authors have completed the ICMJE uniform disclosure form (available at http://dx.doi. org/10.21037/tau-20-998). The authors have no conflicts of interest to declare.

Ethical Statement: The authors are accountable for all aspects of the work in ensuring that questions related to the accuracy or integrity of any part of the work are appropriately investigated and resolved. The local institutional review board (No. RIF.CE: 4882) approved this prospective study, and written informed consent was obtained from all patients. This study was conducted according to the Declaration of Helsinki (as revised in 2013) for clinical studies.

Open Access Statement: This is an Open Access article distributed in accordance with the Creative Commons Attribution-NonCommercial-NoDerivs 4.0 International License (CC BY-NC-ND 4.0), which permits the noncommercial replication and distribution of the article with the strict proviso that no changes or edits are made and the original work is properly cited (including links to both the formal publication through the relevant DOI and the license). See: https://creativecommons.org/licenses/by-nc-nd/4.0/.

\section{References}

1. Engels M, Span PN, van Herwaarden AE, et al. Testicular adrenal rest tumors: current insights on prevalence, characteristics, origin, and treatment. Endocr Rev 2019;40:973-87.

2. Corica D, Bottari A, Aversa T, et al. An unusual epididymal localization of testicular adrenal rest tumor in an adolescent with congenital adrenal hyperplasia. Endocrine 2019;66:695-8.

3. Shin YM. Hepatic adrenal rest tumor mimicking hepatocellular carcinoma. Korean J Hepatol 2010;16:338-41.

4. Claahsen-van der Grinten HL, Duthoi K, Otten BJ, et al. An adrenal rest tumour in the perirenal region in a patient with congenital adrenal hyperplasia due to congenital 3beta-hydroxysteroid dehydrogenase deficiency. Eur J Endocrinol 2008;159:489-91.

5. Chen HD, Huang LE, Zhong ZH, et al. Ovarian adrenal rest tumors undetected by imaging studies and identified at surgery in three females with congenital adrenal hyperplasia unresponsive to increased hormone therapy dosage. Endocr Pathol 2017;28:146-51.

6. Sisto JM, Liu FW, Geffner ME, Berman ML. Paraovarian adrenal rest tumors: gynecologic manifestations of untreated congenital adrenal hyperplasia. Gynecol Endocrinol 2018;34:644-6.

7. Wilkins L, Fleischmann W, Howard JE. Macrogenitosomia precox associated with hyperplasia of the androgenic tissue of the adrenal and death from corticoadrenal insufficiency. 
Endocrinology 1940;26:385-95.

8. Barwick TD, Malhotra A, Webb JA, et al. Embryology of the adrenal glands and its relevance to diagnostic imaging. Clin Radiol 2005;60:953-9.

9. Shima Y, Morohashi KI. Leydig progenitor cells in fetal testis. Mol Cell Endocrinol 2017;445:55-64.

10. Smeets EE, Span PN, van Herwaarden AE, et al. Molecular characterization of testicular adrenal rest tumors in congenital adrenal hyperplasia: lesions with both adrenocortical and Leydig cell features. J Clin Endocrinol Metab 2015;100:E524-30.

11. Yu MK, Jung MK, Kim KE, et al. Clinical manifestations of testicular adrenal rest tumor in males with congenital adrenal hyperplasia. Ann Pediatr Endocrinol Metab 2015;20:155-61.

12. Finkielstain GP, Kim MS, Sinaii N, et al. Clinical characteristics of a cohort of 244 patients with congenital adrenal hyperplasia. J Clin Endocrinol Metab 2012;97:4429-38.

13. Puar T, Engels M, van Herwaarden AE, et al. Bilateral testicular tumors resulting in recurrent Cushing disease after bilateral adrenalectomy. J Clin Endocrinol Metab 2017;102:339-44.

14. Hershkovitz E, Arafat M, Loewenthal N, et al. Combined adrenal failure and testicular adrenal rest tumor in a patient with nicotinamide nucleotide transhydrogenase deficiency. J Pediatr Endocrinol Metab 2015;28:1187-90.

15. Roucher-Boulez F, Mallet-Motak D, Samara-Boustani D, et al. NNT mutations: a cause of primary adrenal insufficiency, oxidative stress and extra-adrenal defects. Eur J Endocrinol 2016;175:73-84.

16. Krone N, Arlt W. Genetics of congenital adrenal hyperplasia. Best Pract Res Clin Endocrinol Metab 2009;23:181-92.

17. Bouvattier C, Esterle L, Renoult-Pierre P, et al. Clinical outcome, hormonal status, gonadotrope axis, and testicular function in 219 adult men born with classic 21-hydroxylase deficiency. A French national survey. J Clin Endocrinol Metab 2015;100:2303-13.

18. Falhammar H, Nordenström A. Nonclassic congenital adrenal hyperplasia due to 21-hydroxylase deficiency: clinical presentation, diagnosis, treatment, and outcome. Endocrine 2015;50:32-50.

19. Alswailem MM, Alzahrani OS, Alhomaidah DS, et al. Mutational analysis of rare subtypes of congenital adrenal hyperplasia in a highly inbred population. Mol Cell Endocrinol 2018;461:105-11.

20. Claahsen-van der Grinten HL, Dehzad F, Kamphuis-van
Ulzen $\mathrm{K}$, et al. Increased prevalence of testicular adrenal rest tumours during adolescence in congenital adrenal hyperplasia. Horm Res Paediatr 2014;82:238-44.

21. Jha S, El-Maouche D, Marko J, et al. Individualizing management of infertility in classic congenital adrenal hyperplasia and testicular adrenal rest tumors. J Endocr Soc 2019;3:2290-4.

22. Tanaka M, Enatsu N, Chiba K, et al. Two cases of reversible male infertility due to congenital adrenal hyperplasia combined with testicular adrenal rest tumor. Reprod Med Biol 2017;17:93-7.

23. Collet TH, Pralong FP. Reversal of primary male infertility and testicular adrenal rest tumors in salt-wasting congenital adrenal hyperplasia. J Clin Endocrinol Metab 2010;95:2013-4.

24. Lottspeich C, Müller-Lisse U, Seiler L, et al. Three cases of testicular adrenal rest tumors in congenital adrenal hyperplasia-A diagnostic and therapeutic challenge. Urology 2019;129:24-8.

25. Tiryaki T, Aycan Z, Hücümenoğlu S, et al. Testis sparing surgery for steroid unresponsive testicular tumors of the congenital adrenal hyperplasia. Pediatr Surg Int 2005;21:853-5.

26. Walker BR, Skoog SJ, Winslow BH, et al. Testis sparing surgery for steroid unresponsive testicular tumors of the adrenogenital syndrome. J Urol 1997;157:1460-3.

27. Claahsen-van der Grinten HL, Otten BJ, Takahashi S, et al. Testicular adrenal rest tumors in adult males with congenital adrenal hyperplasia: evaluation of pituitarygonadal function before and after successful testissparing surgery in eight patients. J Clin Endocrinol Metab 2007;92:612-5.

28. Delfino M, Elia J, Imbrogno N, et al. Testicular adrenal rest tumors in patients with congenital adrenal hyperplasia: prevalence and sonographic, hormonal, and seminal characteristics. J Ultrasound Med 2012;31:383-8.

29. Stikkelbroeck NM, Otten BJ, Pasic A, et al. High prevalence of testicular adrenal rest tumors, impaired spermatogenesis, and Leydig cell failure in adolescent and adult males with congenital adrenal hyperplasia. J Clin Endocrinol Metab 2001;86:5721-8.

30. Yılmaz R, Şahin D, Aghayev A, et al. Sonography and magnetic resonance imaging characteristics of testicular adrenal rest tumors. Pol J Radiol 2017;82:583-8.

31. Stikkelbroeck NM, Suliman HM, Otten BJ, et al. Testicular adrenal rest tumours in postpubertal males with congenital adrenal hyperplasia: sonographic and MR features. Eur Radiol 2003;13:1597-603. 
32. Dogra V, Nathan J, Bhatt S. Sonographic appearance of testicular adrenal rest tissue in congenital adrenal hyperplasia. J Ultrasound Med 2004;23:979-81.

33. Speiser PW, Arlt W, Auchus RJ, et al. Congenital adrenal hyperplasia due to steroid 21-hydroxylase deficiency: an Endocrine Society Clinical Practice Guideline. J Clin Endocrinol Metab 2018;103:4043-88.

34. Engels M, Gehrmann K, Falhammar H, et al. Gonadal function in adult male patients with congenital adrenal hyperplasia. Eur J Endocrinol 2018;178:285-94.

35. Dumic M, Duspara V, Grubic Z, et al. Testicular adrenal rest tumors in congenital adrenal hyperplasia-crosssectional study of 51 Croatian male patients. Eur J Pediatr 2017;176:1393-404.

36. Mouritsen A, Jørgensen N, Main KM, et al. Testicular adrenal rest tumours in boys, adolescents and adult men with congenital adrenal hyperplasia may be associated with the CYP21A2 mutation. Int J Androl 2010;33:521-7.

37. Kim MS, Goodarzian F, Keenan MF, et al. Testicular adrenal rest tumors in boys and young adults with congenital adrenal hyperplasia. J Urol 2017;197:931-6.

38. Nermoen I, Rørvik J, Holmedal SH, et al. High frequency of adrenal myelolipomas and testicular adrenal rest tumours in adult Norwegian patients with classical congenital adrenal hyperplasia because of 21-hydroxylase deficiency. Clin Endocrinol (Oxf) 2011;75:753-9.

39. Kocova M, Janevska V, Anastasovska V. Testicular adrenal rest tumors in boys with 21-hydroxylase deficiency, timely diagnosis and follow-up. Endocr Connect 2018;7:544-52.

40. Reisch N, Flade L, Scherr M, et al. High prevalence

Cite this article as: Corcioni B, Renzulli M, Marasco G, Baronio F, Gambineri A, Ricciardi D, Ortolano R, Farina D, Gaudiano C, Cassio A, Pagotto U, Golfieri R. Prevalence and ultrasound patterns of testicular adrenal rest tumors in adults with congenital adrenal hyperplasia. Transl Androl Urol 2021;10(2):562-573. doi: 10.21037/tau-20-998 of reduced fecundity in men with congenital adrenal hyperplasia. J Clin Endocrinol Metab 2009;94:1665-70.

41. Claahsen-van der Grinten HL, Sweep FC, Blickman JG, et al. Prevalence of testicular adrenal rest tumours in male children with congenital adrenal hyperplasia due to 21-hydroxylase deficiency. Eur J Endocrinol 2007;157:339-44.

42. Claahsen-van der Grinten HL, Otten BJ, Sweep FC, et al. Testicular tumors in patients with congenital adrenal hyperplasia due to 21-hydroxylase deficiency show functional features of adrenocortical tissue. J Clin Endocrinol Metab 2007;92:3674-80.

43. Lottrup G, Nielsen JE, Skakkebæk NE, et al. Abundance of DLK1, differential expression of CYP11B1, CYP21A2 and MC2R, and lack of INSL3 distinguish testicular adrenal rest tumours from Leydig cell tumours. Eur J Endocrinol 2015;172:491-9.

44. Wang Z, Yang Z, Wang W, et al. Diagnosis of testicular adrenal rest tumors on ultrasound: a retrospective study of 15 cases report. Medicine (Baltimore) 2015;94:e1471.

45. Chihaoui M, Kanoun F, Chaker F, et al. Testicular adrenal rest tumours in young adult males with congenital adrenal hyperplasia: prevalence and impact on testicular function. Andrologia 2016;48:45-50.

46. Mnif MF, Kamoun M, Mnif F, et al. Long-term outcome of patients with congenital adrenal hyperplasia due to 21-hydroxylase deficiency. Am J Med Sci 2012;344:363-73.

47. Cabrera MSL, Vogiatzi MG, New MI. Long Term outcome in adult males with classic congenital adrenal hyperplasia. J Clin Endocrinol Metab 2001;86:3070-8. 